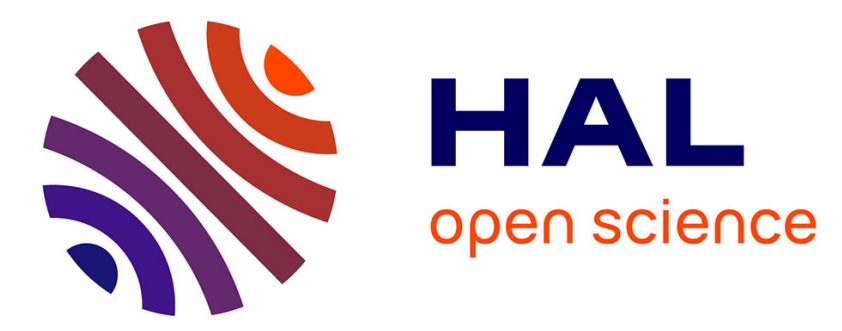

\title{
Observation of period-doubling dynamics of modulation instability in uniform and dispersion oscillating fiber-ring cavities
}

François Copie, Florent Bessin, Matteo Conforti, Alexandre Kudlinski, Stefano Trillo, Arnaud Mussot

\section{To cite this version:}

François Copie, Florent Bessin, Matteo Conforti, Alexandre Kudlinski, Stefano Trillo, et al.. Observation of period-doubling dynamics of modulation instability in uniform and dispersion oscillating fiber-ring cavities. Nonlinear Photonics, Jul 2018, Zurich, Switzerland. NpW1C.4, 10.1364/NP.2018.NpW1C.4 . hal-02393201

\section{HAL Id: hal-02393201 \\ https://hal.science/hal-02393201}

Submitted on 6 Dec 2019

HAL is a multi-disciplinary open access archive for the deposit and dissemination of scientific research documents, whether they are published or not. The documents may come from teaching and research institutions in France or abroad, or from public or private research centers.
L'archive ouverte pluridisciplinaire HAL, est destinée au dépôt et à la diffusion de documents scientifiques de niveau recherche, publiés ou non, émanant des établissements d'enseignement et de recherche français ou étrangers, des laboratoires publics ou privés. 


\title{
Observation of period-doubling dynamics of modulation instability in uniform and dispersion oscillating fiber-ring cavities
}

\author{
François Copie, ${ }^{1}$ Florent Bessin, ${ }^{1}$ Matteo Conforti, ${ }^{1}$ Alexandre Kudlinski, ${ }^{1}$ Stefano Trillo, $^{2}$ Arnaud Mussot ${ }^{1}$ \\ ${ }^{1}$ Univ. Lille, CNRS, UMR 8523-PhLAM-Physique des Lasers Atomes et Molécules, F-59000 Lille, France \\ ${ }^{2}$ Department of Engineering, University of Ferrara, Via Saragat 1, 44122 Ferrara, Italy \\ arnaud.mussot@univ-lille1.fr
}

\begin{abstract}
We provide a direct observation of a period-doubling phenomenon associated to the modulation instability in both uniform and dispersion oscillating passive fiber ring cavities. This has been done by performing roundtrip-to-roundtrip measurements of the temporal pattern generated through the instability. (c) 2018 The Author(s)

OCIS codes: (190.4370) Nonlinear optics, fibers; (190.3100) Instabilities and Chaos; (230.5750) Resonators
\end{abstract}

\section{Introduction}

In a seminal work, Ikeda [1] predicted period doubling (P2) process before reaching chaos in passive cavities. Later on, in the first experimental evidence of modulation instability (MI) in normal dispersion fiber ring cavities, Coen et al. [2], developed a theoretical model to describe P2 regime associated with MI and provided an experimental evidence in the spectral domain. Indeed, different frequency shifts (phase matching) are associated to P1 and P2 regimes which allows to get a clear signature in the spectral domain. But the most striking feature occurs in the time domain where P1 regime leads to periodic temporal structures in phase round to round trip while P2 one is characterized by a $\pi$ shift at each round trip. This shift originates from the negative value of the eigenvalue associated to MI gain at the anti-resonance of the system [2]. The occurrence of P2 regime is not peculiar to homogenous cavities but can also be observed in dispersion modulated cavities, where different instability processes can take place, (i) the Turing one, due to periodic boundary conditions of the cavity and (ii) the Faraday one, due to periodic dispersion variations [3]. Turing instability is associated with P1 regime while the parametric nature of Faraday instabilities leads to the onset of many resonances (Arnold tongs) alternating P1 and P2 regimes [4].
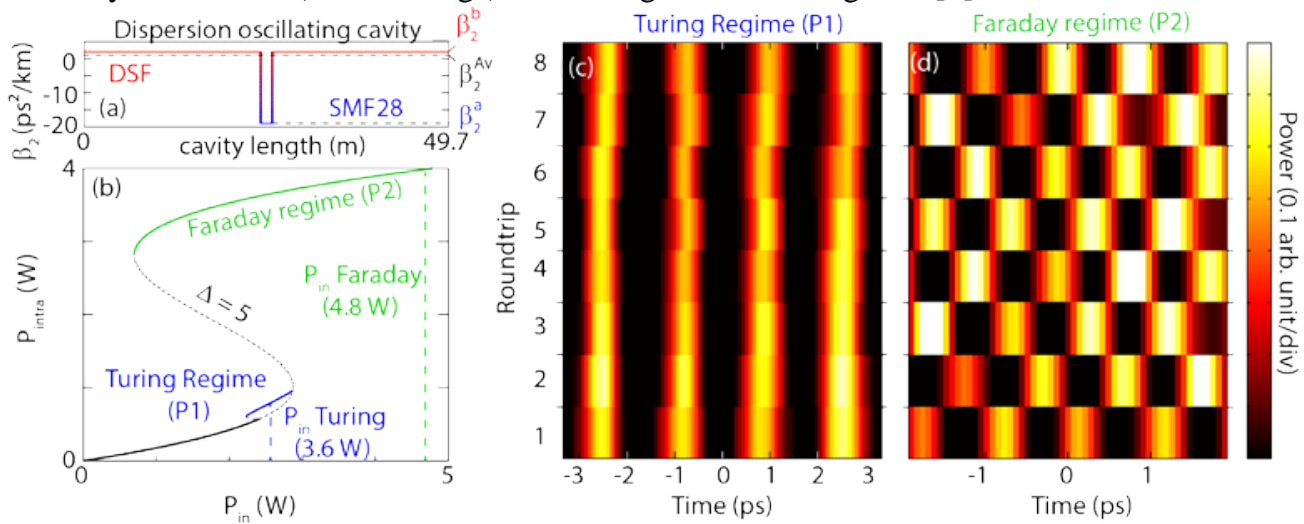

Fig. 1. Experimental results in a dispersion oscillating cavity. (a) Design and longitudinal dispersion profile of the modulated fiber-ring cavity. (b) Bistable response of the cavity for a normalized detuning $\Delta=5$. Blue (green) line denotes the Turing (Faraday) branch. (c) 2D color plot of the evolution of temporal traces recorded at the output of the cavity in the Turing regime (P1) and (d) in the Faraday regime (P2).

In this communication, we provide, to our knowledge, the first direct observation of the P1/P2 regimes in the time domain induced by modulation instability in a nonlinear optical resonator. We observed them in a uniform cavity by either working close to its resonance (P1) or anti-resonance (P2), as well as in a dispersion oscillating cavity, by either exciting Turing (P1) or Faraday instability (P2). To do so, we implemented a temporal magnifier (time lens system) to resolve the temporal structures of a few picosecond periods delivered by the cavity. This allows us to record temporal traces on a window spanning a few tens of $\mathrm{ps}$ with a resolution of $\approx 300 \mathrm{fs}$. 


\section{Dispersion oscillating cavity}

We fabricated a dispersion modulated fiber cavity by splicing a 48-m-long dispersion shifted fiber (DSF, $\beta_{2}=2 \mathrm{ps}^{2} / \mathrm{km}$ at the pump wavelength-1545 nm-) to a 90/10 standard SMF28 coupler (Fig. 1(a)). When the cavity is pumped in the bistable regime ( $\Delta=5$, Fig. 1(b)), Turing instability is susceptible to occur on the lower branch while Faraday instability occurs over the upper branch [3]. First, we drove the cavity with pulses whose peak power makes the system experience the Turing instability. Corresponding time traces recorded after the system reached a stable state are plotted in Figs. 1(c). We observe the existence of a sustained periodic modulation in the form of 4 vertical bright stripes of 1.6 ps period, which is symptomatic of a P1 dynamics. Secondly, we increased the pump power so that the system switches to the upper branch of the bistable cycle and experiences now the Faraday instability. As can be seen in Fig. 1 (d), the result strikingly differs from the previous one. The 2D color plot now takes the peculiar form of a check pattern: the temporal trace alternates between two out-of-phase modulated waveforms at each roundtrip, that is characteristic of the P2 regime. The structure period is also different and equals to $950 \mathrm{fs}$. Numerical simulations (not shown here) are in pretty good agreement with these experiments.

\section{Uniform cavity}

We fabricated a uniform fiber cavity made with a dispersion shifted fiber $\left(\beta_{2}=9.3 \mathrm{ps}^{2} / \mathrm{km}\right.$ at $\left.1545 \mathrm{~nm}\right)$ and a $90 / 10$ coupler from the same fiber (Fig. 1(a)). The cavity is pumped close to its resonance to excite MI in the P1 regime (cavity detuning, $\delta=0.92 \mathrm{rad}$, see inset in Fig. 2(a) and cavity response in Fig. 2(b)). Output temporal traces are shown in Fig. 2 (d) for 6 successive round trips.
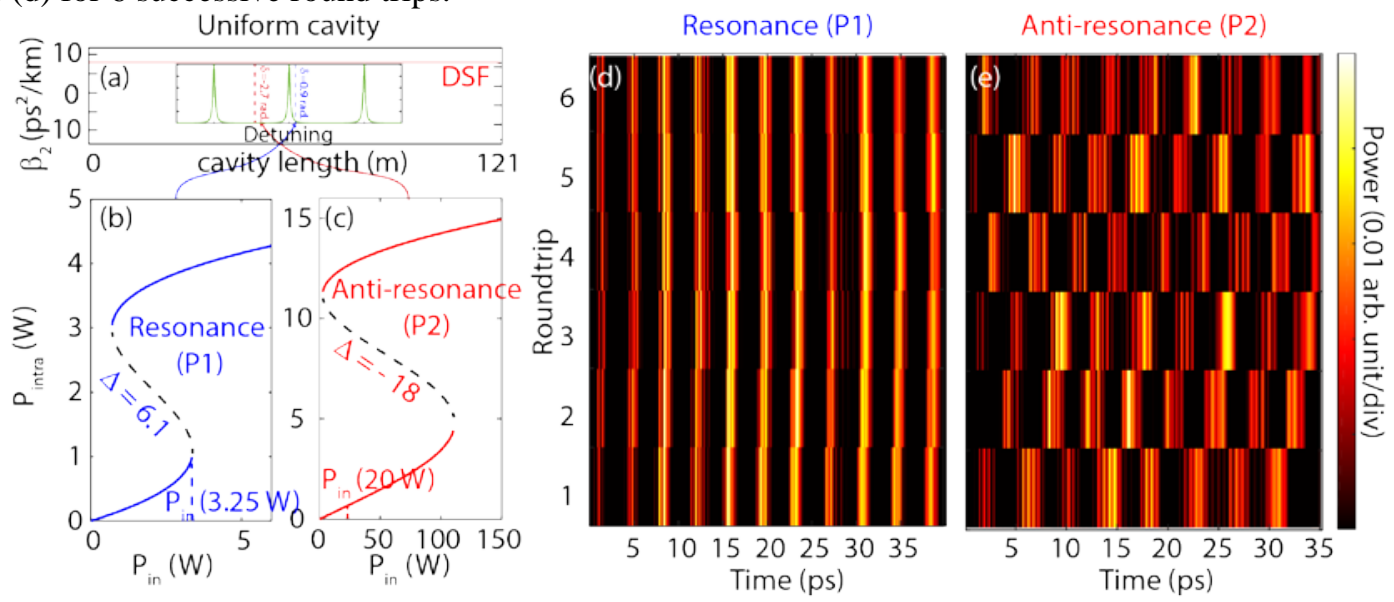

Fig. 2. Experimental results in a uniform cavity. (a) Uniform fiber-ring cavity (inset: transfert function of the cavity). (b) Bistable response of the cavity for a normalized detuning $\Delta=6.1$ (close to the resonance, P1) and (c) close to the anti resonance (P2, $\Delta=-18$ ). 2D color plot of the evolution of temporal traces recorded at the output of the cavity close to (d) the resonance (P1) and (e) the anti-resonance (P2).

As previously, straight strips are observed (period of 3.75 ps) that is characteristic to P1 regime. By tuning the detuning close to the anti-resonance of the cavity (cavity detuning, $\delta=-2.7$ rad, see inset in Fig. 2(a) and cavity response in Fig. 2(c)), temporal output traces look now as a check pattern that is characteristic of P2 regime. The period of the pattern is different from the P1 regime and equals to 4.3 ps. Numerical simulations (not shown here) are in pretty good agreement with these experiments.

\section{Conclusion}

By means of roundtrip-to-roundtrip ultrafast temporal measurements, we observed experimentally for the first time to our knowledge the temporal signature of the P2 regime occurring in passive fiber ring cavities. We report it in two different cavity architectures, one uniform cavity and one dispersion oscillating cavity, illustrating the widespread interest of this fundamental nonlinear dynamical effect to any passive nonlinear resonators.

[1] Ikeda, K. "Multiple-valued stationary state and its instability of the transmitted light by a ring cavity system”. Optics Communications 30 , 257-261 (1979).

[2] Coen, S. and Haelterman, M. “Modulational Instability Induced by Cavity Boundary Conditions in a Normally Dispersive Optical Fiber”. Phys. Rev. Lett. 79, 4139-4142 (1997).

[3] F. Copie, M. Conforti, A. Kudlinski, A. Mussot, and S. Trillo, "Competing Turing and Faraday Instabilities in Longitudinally Modulated Passive Resonators,” Phys. Rev. Lett. 116, 143901 (2016).

[4] Conforti, M., Copie, F., Mussot, A., Kudlinski, A. \& Trillo, S.” Parametric instabilities in modulated fiber ring cavities”. Opt. Lett., OL 41, 5027-5030 (2016). 